\title{
$\begin{array}{ll}\text { Research Square } & \text { Preprints are preliminary reports that have not undergone peer review. }\end{array}$ or referenced by the media as validated information. \\ Prehatching Temperatures Drive Interannual Cohort Differences In Great Tit Metabolism
}

Juli Broggi ( $\nabla$ julibroggi@gmail.com )

Estación Biologica de Doñana, CSIC https://orcid.org/0000-0002-1706-4014

\section{Esa Hohtola}

University of Oulu: Oulun Yliopisto

Kari Koivula

Oulun Yliopisto

Seppo Rytkönen

Oulun Yliopisto

Jan-Åke Nilsson

Lund University: Lunds Universitet

\section{Research Article}

Keywords: Basal metabolic rate, Breeding vs wintering, Carry-over, Early developmental conditions, Transgenerational plasticity

Posted Date: November 8th, 2021

DOI: https://doi.org/10.21203/rs.3.rs-875558/v1

License: (c) (i) This work is licensed under a Creative Commons Attribution 4.0 International License.

Read Full License 


\section{Abstract}

Basal metabolic rate (BMR) constitutes the lowest metabolic rate in a resting animal and is therefore considered to reflect the energetic cost of maintenance in endotherms. BMR is a reversible plastic trait that changes with environmental and ecological circumstances, albeit being heritable and susceptible to selection. Inter-individual variation within populations of small birds is substantial, and while many of the drivers of such variation have been identified, many remain unexplained. Some of the variation can be ascribed to cohort effects, indicating non-reversible developmental plasticity that will shape the adult metabolic phenotype. We studied winter BMR variation of juveniles over a fifteen-year period in a wild population of great tits Parus major at the northern border of their distribution.

Here we show for the first time that winter BMR in cohorts of great tits changes among winters as a response to minimum ambient temperatures experienced early in life, during the prehatching period. This developmental plasticity might be adaptive if temperatures experienced by growing embryos would metabolically prime them to an environment that they will likely encounter in future life. However, in line with a more unpredictable future climate, the risk of phenotype-environment mismatch is likely to lead to certain cohorts being poorly adapted to prevailing winter conditions, resulting in wider annual fluctuations in population size.

\section{Introduction}

Small birds acclimatize to winter conditions by improving their cold-endurance and thermogenic capacity, which correlates with a seasonal increase of internal energy reserves and basal metabolic rate (BMR) (Swanson 2010). BMR constitutes the lowest metabolic rate in a resting animal and is thereby considered to reflect physiological maintenance costs. In general, selection should keep the maintenance costs as low as possible (Swanson et al. 2017), although there might be individual variation in the optimal level of investment in maintenance (Petit and Vézina 2014a). Maintenance costs constitute a major part of energy expenditure expended energy during winter nights in resident birds particularly at high latitudes (Broggi et al. 2004), which often correlates with the thermogenic capacity in these bird species (Broggi et al. 2004; Swanson 2010; McKechnie and Swanson 2010). However, this relation is not universal as BMR and maximal energy expenditure for thermogenesis, representing cold endurance and thermogenic capacity, lack a functional link as they are influenced by different physiological systems (Petit et al. 2013; Petit and Vézina 2014a). Variation in BMR is mostly influenced by the size and cellular activity of organs responsible for energy acquisition and processing of food, whereas variation in maximal energy expenditure for thermogenesis is primarily influenced by the overall size, size of individual muscle fibres and cellular activity of the pectoralis muscles, which are ultimately responsible for shivering thermogenesis (Jimenez and Williams 2014; Petit and Vézina 2014b). Although, the size and activity of these systems often vary in concert in relation to season (Zheng et al. 2014a), BMR has been documented to respond faster to changes in ambient temperature (Dubois et al. 2016) and show a different temporal adjustment to seasonal changes (Petit et al. 2013) than maximal energy expenditure for thermogenesis. Furthermore, both BMR (Rønning et al. 2016; Nilsson and Nilsson 2016) and maximal 
energy expenditure for thermogenesis (Petit et al. 2017; Latimer et al. 2018) have been documented to influence winter survival.

Thus, intra-specific variation in BMR is primarily a consequence of variation in size and activity of organ systems such as liver, kidney, heart and the alimentary tract (Wiersma et al. 2012; Swanson et al. 2017). However, variation in BMR also depends on both genetic factors and phenotypic plasticity. BMR remains individually consistent (Versteegh et al. 2008; Broggi et al. 2009) with moderate but significant heritability in studies of passerine birds (Rønning et al. 2007; Nilsson et al. 2009; Tieleman et al. 2009; Bushuev et al. 2012; Mathot et al. 2013). However, it is also a labile trait (i.e. phenotypically flexible) that can change reversibly with environmental and ecological circumstances (see McKechnie (2008) and Swanson (2010) for reviews). Previous intra-specific studies have reported BMR plasticity within individuals, resulting in variation among populations in responses to prevailing conditions, including photoperiod (Broggi et al. 2019) and ambient temperature (e.g. Swanson and Olmstead 1999; McKechnie et al. 2007; Petit and Vézina 2014a; Broggi et al. 2019). Ambient temperature also proved to be the main driver explaining interspecific variation in BMR in rodents (Naya et al. 2013).

However, after statistically accounting for responses to prevailing environmental conditions during winter, differences in BMR between winters often remain significant (Broggi et al., 2019). This inter-annual variation has been interpreted as originating from stochastic variation (Bouwhuis et al. 2011), although it might also be indicative of cohort effects (Swanson and Olmstead 1999; Broggi et al. 2019). Conditions during early development can have a crucial influence on the adult phenotype (Reid et al. 2003), either influencing the developing offspring directly, or through indirect effects mediated through parental performance i.e. transgenerational plasticity (Beaman et al. 2016). Thus, shared environmental conditions during development may result in phenotypic uniformity among young in synchronously breeding populations. Such cohort effects have been demonstrated in passerines, for example in the redbilled chough Pyrrhocorax pyrrhocorax (Reid et al. 2003), but primarily in three well-studied ungulate populations (Rose et al. 1999; Forchhammer et al. 2001; Douhard et al. 2013), with potential effects on population dynamics (Lindström and Kokko 2002).

Although, cohort effects based on variation in early-life conditions have mostly been reported as variations in growth rate or survival probability, some underlying mechanistic traits have also been suggested, e.g. variation in telomere length (Fairlie et al. 2016). Thus, many morphological and physiological traits may be susceptible to non-reversible developmental plasticity, which could predetermine, for example, the adult metabolic phenotype because of conditions experienced during specific developmental stages (West-Eberhard 2003; McKechnie and Swanson 2010). In avian systems, ambient temperature stands out among environmental drivers, as it can influence the developing offspring directly during the pre- and post-hatching stages, and indirectly as influenced by parental performance during egg laying, incubation and brooding (Careau et al. 2014; Beaman et al. 2016). However, the long-term consequences of such early conditioning, and the precise effect of the environmental/physiological drivers remain poorly understood, particularly in wild animal populations. 
We studied the among-year variation in winter BMR of first-year, wild great tits Parus major, while controlling for within-winter environmental conditions to reveal potential cohort effects. Cohort effects might be particularly influential in short-lived, small passerines, as these populations are composed by a large proportion of first-year birds. We tested the influence of ambient temperatures during different developmental stages and predicted that the early-life conditions would affect the standardized BMR during their first winter. First, cold ambient temperatures during the egg laying stage may reduce parental investment in eggs (Ojanen 1983). Smaller eggs, or eggs produced by females in poor condition have been found to contain higher levels of androgens (Groothuis et al. 2005), resulting in increased growth rate and begging behaviour (Reed and Clark 2011), with a concomitant increase in nestling metabolism (Bachman and Chappell 1998; Jimenez 2018). Second, suboptimal incubation temperatures have been shown to increase BMR of developing nestlings (Nord and Nilsson 2011). Finally, cold ambient temperatures during the nestling phase may reduce parental investment and induce nestling developmental stress, leading to increased BMR (Criscuolo et al. 2008; Careau et al. 2014). If these increases in nestling and juvenile metabolism are irreversible, we predict that cold ambient temperatures during the egg laying, incubation and nestling feeding stages, respectively, will increase adult BMR.

\section{Materials And Methods}

Study area and birds

We measured winter BMR of wild, first-year great tits in Oulu (Finland) $\left(65^{\circ} \mathrm{N}, 25^{\circ} 30^{\prime} \mathrm{E}\right)$, over a period of 11 winters from 1999-2000 until 2005-2006, 2007-2008 and from 2011-2012 until 2013-2014 from midOctober to mid-April (median 16 January). The Oulu study area is composed of boreal forests dominated by conifers, Scots pine Pinus sylvestris and spruce Picea abies, and diverse birch Betula sp. species. Winter conditions in the Oulu area are influenced by the high latitude, with minimum temperatures on capture days ranging from $-32.0^{\circ} \mathrm{C}$ to $+7^{\circ} \mathrm{C}$ and day lengths during the peak of mid-winter of $3.5 \mathrm{~h}$.

The great tit is a small-sized passerine $(19.2 \mathrm{~g} \pm 1.53)$ that inhabits temperate forests across Eurasia. In our study area, great tits start egg laying during mid-May, females incubate alone while both parents feed the nestlings. During winter months, great tits invariably move from the breeding territories and join loose flocks near human settlements. The species is actively spreading northwards through Fennoscandia, with the studied population in the Oulu region being close to the northernmost margin of the distribution (Valkama et al. 2011). Boreal conditions are suboptimal for the species, which result in low and temporally fluctuating juvenile survival rates likely as a consequence of harsh winter conditions (Karvonen et al. 2012). Additionally, as many other passerine species at northern latitudes, the Oulu population is time-constrained by the short breeding season, which promotes synchronous breeding (Pakanen et al. 2016).

Birds were captured in the vicinity of the University campus soon before dusk at baited funnel traps and weighed (to the nearest $0.1 \mathrm{~g}$ ). Since few individuals in our winter population are recaptures from the studied breeding population located $10 \mathrm{~km}$ to the north, birds were sexed and aged (as first-year or older) 
by plumage characteristics (Jenni and Winkel 1994), individually marked and brought to the university facilities for BMR measurements (see below). In total we captured 117 first-year individuals from which we obtained 160 BMR measurements, with up to 5 re-captures of the same individual within a winter. During each winter, we measured between 2 and 29 first-year individuals (Fig. 1). Fifteen first-year great tits were also captured in subsequent winters (12 individuals during two and 3 during three consecutive winters) making it possible to calculate between-year repeatability. More details on the study area and trapping procedures are provided elsewhere (Broggi et al. 2004).

Table 1

Final model with significant factors explaining first-year great tit BMR (ml $\mathrm{O}_{2} / \mathrm{min}$ ) during winter. Min temperature refers to the average minimum temperature the week before the metabolic rate measurements. Winter refers to the winter of measurement. Continuous variables are presented with estimate $( \pm \mathrm{SE})$ and all variables with F-value, df and P-value. $\mathrm{N}=117$ individuals.

\begin{tabular}{|lllll|}
\hline Factor & Estimate $( \pm \mathrm{SE})$ & F-value & $\mathbf{d f}$ & $\mathbf{P}$ \\
\hline Date & $-0.00093(0.00027)$ & 12.0 & 1,122 & 0.0007 \\
\hline Min temperature & $-0.00653(0.00196)$ & 11.1 & 1,145 & 0.0011 \\
\hline Winter & & 4.35 & 10,129 & $<0.0001$ \\
\hline Body mass & $0.04463(0.00790)$ & 31.9 & 1,122 & $<0.0001$ \\
\hline
\end{tabular}

Metabolic measurements

BMR is defined as the average minimal oxygen consumption under post absorptive digestive conditions during the resting phase of the daily cycle of non-growing, non-reproductive endotherms at thermoneutrality (McNab 1997). Thus, BMR was measured as the lowest 5 -minute running averages of oxygen consumption during the night, in an open-circuit respirometer in a dark climate cabinet, at a constant temperature of $25^{\circ} \mathrm{C}$ (mean \pm S.D. for a typical night: $24.8 \pm 1.65$ ) that is within the species thermoneutral zone (Reinertsen and Haftorn 1986). In short, outdoor dry air was pushed through four independent mass-flow controllers (FMA-A2407, Omega Engineering, Inc. (USA)) at $300 \mathrm{ml} / \mathrm{min}$ into plexiglass metabolic chambers $(1,6 \mathrm{~L})$ containing an individual bird. Constantly flowing incoming and outcoming air was scrubbed for $\mathrm{CO}_{2}$ and $\mathrm{H}_{2} \mathrm{O}$, with sodalime and silica scrubbers, then conveyed through a multiplexer that directed air from each channel in turns of $12 \mathrm{~min}$ to the Oxygen analyzer (S-3A Ametek (USA)). Each cycle lasted an hour and included a fifth channel with reference air to control for the analyzer bias, making it possible to measure a maximum of four birds simultaneously per night. Recorded data were visually inspected and converted to $\mathrm{mL} \mathrm{O}_{2} / \mathrm{min}$ following Hill (1972). Most measurement sessions (80 \%) started between 15:00 and 18:00 (range 15:00-21:00) and BMR estimates (the lowest 5-minute running averages of oxygen consumption) were invariably recorded between midnight and 04:00, ensuring postabsorptive state of all individuals (own unpubl. data). After the overnight measurements, birds were released at the point of capture. All procedures were conducted in agreement with the local and national ethical committees. 
In a first set of analyses, we studied the effects of the regional and local winter environment on winter BMR of the first-year birds. Date (October $1 s t=1$ ) was included as a linear factor to control for any somatic or physiological effects on BMR, accumulated progressively since the winter start (Swanson 2010). Furthermore, we also included day length (time between sun rise and sun set) to represent the time available for feeding and night-time forced fasting (Carey and Dawson 1999; Broggi et al. 2019). We included the North Atlantic Oscillation (NAO) index (obtained from https://www.cpc.ncep.noaa.gov/products/precip/CWlink/pna/nao.shtml) as a measure of large-scale (regional) climatic effects (calculated as the monthly average prior to each individual's capture date). Positive NAO index is generally associated with milder and moister winter conditions at higher latitudes in Western Europe. Furthermore, to evaluate the effect of local conditions we incorporated the average minimum ambient temperature $\left({ }^{\circ} \mathrm{C}\right)$ that reflects thermoregulatory costs, and the atmospheric pressure (mBAR) that can be used as a proxy of precipitation (Carey and Dawson 1999). These local conditions were estimated for each capture dates over three different time periods, viz. immediate (day of capture), previous short-term (daily average of the week before capture) and previous long-term (daily average of the month before capture) environmental conditions from the date of capture.

In a second set of analyses, we studied the effect of ambient temperature during early development on the metabolic phenotype of great tits. We estimated population average breeding parameters from the neighbouring nest-box study population (10 km north of the winter trapping area) that has been continuously monitored over decades (Karvonen et al. 2012; Pakanen et al. 2016; Vatka et al. 2016). Here we used annual average date of first egg (77-141 estimates per year), clutch size (75-148 clutches per year) and hatching date (63-118 estimates per year) to define three developmental periods for each year of the study. The periods considered were defined as: Egg laying: laying of the first egg + clutch size; Incubation: from 14 days previous to hatching date until hatching date (from estimates of the incubation length in the same population see Pakanen et al. (2016)); Nestling provisioning: hatching date +21 days (based on an average nestling period of 18 days from estimations in the same population (Orell and Ojanen 1983) plus three days as fledglings when they potentially are susceptible to environmental conditions. For these time periods, we extracted daily average and minimum temperatures for the year when each measured first-year great tit was born. Atmospheric pressure and ambient temperatures were obtained from the climate station Oulu Linnanmaa situated $4 \mathrm{~km}$ south of the trapping area.

Statistical analyses

All analyses were performed in SAS Enterprise Guide 7.1 (SAS Institute Inc., Cary, NC, USA). To evaluate the effects of the minimum ambient temperature $\left({ }^{\circ} \mathrm{C}\right.$ ) and atmospheric pressure (mBAR), we first tested which of the three time periods (immediate, previous short-term and previous long-term) that best explained the observed variation. Since the three measures of temperature and atmospheric pressure are interdependent, the effects were included separately together with body mass as a covariate, and their fit was evaluated by Akaike's information criterion (AIC). The period with the best fit for both the average 
minimum temperature and for the average atmospheric pressure was the week before BMR measurements (previous short-term) and these two factors were included in the model of within-winter effects on BMR.

In a first set of analyses, we evaluated the effect of intrinsic factors of the individuals (sex and body mass) together with prevailing environmental conditions (NAO index, date, day length, winter, minimum temperature, and atmospheric pressure) on winter BMR. We used general linear mixed models with winter (winter 1999-2000 $=1)$ and sex $(0=$ male and $1=$ female) included as categorical predictors and individual identity as a random factor, while body mass, NAO index, date, day length as well as short-term average minimum temperature and atmospheric pressure were considered as continuous predictors. To accommodate different effects of day length in early and late parts of the winter season, we also included the interaction between date and day length. The full model was reduced in a stepwise fashion until only significant variables remained.

In a second set of analyses, we extracted yearly standardized means from the final model of intrinsic and winter environmental factors to study among-winter variation in BMR. Thus, we used the predicted values from this final model explaining within-winter sources of variation as the dependent variable in the following analyses. To investigate the potential influence of ambient temperature during early life on the winter metabolic phenotype of first-year great tits, we performed two sets of analyses; one with the average daily temperature during the egg laying, the incubation and the nestling phases, and one where average mean temperature was exchanged for average minimum temperature. Variance inflation factors (VIFs) for the temperature estimates was only moderately collinear as VIFs were $<2.5$, thus below the level VIF > 3 when collinearity could bias analyses (Zuur et al. 2010). These two models were fitted with the temperature measurements during the three developmental periods and average laying date as covariates, reduced in a step-wise fashion until only significant factors remained and the two sets of models were then compared using AIC. Models were estimated by REML and individual was included as a random factor. We used the autoregressive covariance structure and estimated degrees of freedom by the Satterthwaite method. Sequential reintroduction of each eliminated main effect back into the final model never improved the fit (as determined by AIC). Full models and parameters removed are presented with the corresponding values when removed from the model (Table S1 and S2).

We also tested for a potential association between average minimum temperature during spring and the average daily minimum temperatures during January and February the succeeding winter. Repeatabilities were calculated by a one-way ANOVA according to Lessells and Boag (1987). When individuals were measured more than once within a winter, we selected one estimate per year to achieve a time period between measurements as close to 365 days as possible (cf. Broggi et al. 2009; Cortés et al. 2015). All Pvalues are two-tailed. All continuous variables and model residuals fulfilled the requirements of normality.

\section{Results}


Average winter BMR was $1.28 \pm 0.18 \mathrm{~mL} \mathrm{O} / \mathrm{min}$. Of the factors that could be predicted to have a direct effect on the winter BMR of first-year great tits (viz. intrinsic, climatic and date factors), four explained a significant part of the variation in BMR (Table 1). BMR decreased linearly as winter progressed (Fig. S1) and increased with decreasing minimum ambient temperatures (Fig. S2), while it increased with body mass (Table 1). However, even when accounting for these sources of variation, we still found substantial among-winter differences not explained by the other explanatory variables (Table 1; Fig. 1). To account for some of the inter-annual variation in BMR, independently from winter-specific environmental conditions, we explored the effect of conditions experienced in earlier life stages, i.e. during egg laying, incubation and nestling provisioning. We found that only temperatures during the incubation period significantly explained this inter-annual variation in population BMR of first-year birds in both sets of temperature models (average temperature: $F_{1,9}=11.4, p=0.0081$; minimum temperature: $F_{1,9}=17.9, p=$ 0.0022). Judging from AIC, the model with minimum temperature performed better than the one with average temperature $(\triangle A I C=2.8)$, the two models explaining 67 and $56 \%$, respectively, of the variation in BMR of first-year individuals among winters (Fig. 2). Furthermore, we found a moderate ability for the average minimum temperature during incubation to predict the average minimum winter temperature during the following months of January and February at our study site (linear regression: $F_{1,14}=6.59, p=$ $0.022, R^{2}=0.32$, slope $=+0.89$ ). Temperatures during the other two time periods (i.e. egg laying and nestling provisioning periods) had no significant effect on winter BMR (Table S2).

Furthermore, since some of the yearly estimates were based on few BMR measurements (Fig. 1), we restricted the analyses of the relation between winter BMR and average ambient temperatures during the different breeding stages, to years in where at least 7 first-year individuals were measured ( $N=8$ winters). As in the analyses of the full data-set, average minimum ambient temperature best explained our variation in winter BMR and this temperature during the incubation phase was the only factor significantly related to BMR of the first-year great tits during winter (estimate $\pm S E:-0.030 \pm 0.0084, F_{1,6}=$ $12.7, p=0.012$ ). In addition, BMR was significantly repeatable within individuals between winter seasons $\left(r=0.454, F_{14,18}=2.83, p=0.020\right)$.

\section{Discussion}

The winter BMR of first-year great tits was affected by both reversible and non-reversible environmental effects, especially in relation to ambient temperature. Individual winter BMR increased with decreasing minimum temperatures, suggesting a reversible effect of ambient temperature that has also been commonly reported in other studies of tits (Bouwhuis et al. 2011; Petit and Vézina 2014a; Broggi et al. 2019). However, most importantly, when the variation due to winter conditions were accounted for, the minimum ambient temperatures experienced early in life, during the incubation period, explained a significant part of the remaining variation in winter BMR. Low minimum ambient temperatures during incubation resulted in wintering first-year individuals with higher BMR than predicted from e.g. ambient minimum temperatures during the winter (Fig. 2). Furthermore, we found a significant repeatability of BMR between winters $(r=0.454)$ similar to previous between-year repeatabilities calculated on a larger 
data-set from the same population ( $r=0.533$, Broggi et al. 2009). Thus, first-year birds, incubated during cold conditions, seem to be primed to a high BMR irrespective of encountered winter conditions later in life, as opposed to those incubated during warm springs. This permanent early effect on BMR suggests a form of non-reversible plasticity, due to either a direct effect of ambient temperature on developing embryos, or an indirect effect modulated by maternal incubation behaviour that resulted in long-term effects on the adult metabolic phenotype. We acknowledge that this is a descriptive study and thereby has limited causal predictive power. Experimental incubation of fertilized eggs at different temperatures and monitoring metabolic parameters in subsequent winters would be necessary to provide such a causal link. This was done in blue tits (Nord and Nilsson 2011), although the species has a too low recruitment rate to be able to test the long-term metabolic consequences of different incubation temperatures (Nord and Nilsson 2016).

Incubation entails high levels of heat loss (Mertens 1980) leading to considerable metabolic costs for avian parents in cold environments (Thomson et al. 1998) that can be traded-off against investment in future reproduction (Ardia and Clotfelter 2007). Especially in species with uniparental incubation as the great tit, egg temperature is necessarily influenced by prevailing ambient temperature (Ardia and Clotfelter 2007; Ardia et al. 2009). In cold conditions, reduction in egg temperature results in longer incubation periods (Järvinen 1990; Martin et al. 2018), even under increased incubation effort (Nord et al. 2010). Furthermore, suboptimal egg temperatures during incubation are known to impinge on nestling phenotype, with short-term effects on the thermogenic costs of thermoregulation (DuRant et al. 2012) and BMR (Nord and Nilsson 2011; Wada et al. 2015) as well as on survival in captivity (Berntsen and Bech 2016). We found evidence for long-term effects of varying temperatures during development in the egg on adult metabolic phenotype that seem to be non-reversible judging from the significant repeatability between winters. Thus, the thermal environment experienced by the embryos during incubation could promote a transgenerational plastic effect leading to metabolic differences among cohorts.

Conditions experienced during early development, either during the nestling phase (Verhulst, Holveck and Riebel 2006; Criscuolo et al. 2008) or during the embryonic stage (Ben-Ezra et al. 2017; this study), may pre-determine the adult metabolic phenotype. There is an unfortunate paucity in studies on potential mechanisms for such a priming of the metabolic phenotype (Nord and Giroud 2020). One candidate mechanism might be temperature-induced epigenetic adaptations, e.g. varying levels of DNA methylation as found in zebra finches exposed to different ambient temperatures during early life (Sheldon et al. 2020). A potential specific mechanism might be epigenetic effects during the ontogeny of the hypothalamus-pituitary-thyroid (HPT) axis (Tzschenke 2008; Loyau et al. 2014; Ruuskanen et al. 2021). The target for epigenetic alterations may be the production of thyroid hormones (especially triiodothyronine, $\mathrm{T}_{3}$ ), demonstrated to increase in cold environments (Zheng et al. 2014b). Increased levels of $T_{3}$ is known to increase thermogenesis and to simultaneously increase BMR (Vézina et al. 2009; Zheng et al. 2014b; Ruuskanen et al. 2021). This mechanism is based on alterations during ontogeny, i.e. during the incubation stage. The fact that we did not find any effects of ambient temperature during the 
egg laying and nestling stages on adult BMR is compatible with this mechanism. Previous demonstrations of environmental effects originating during the egg laying and nestling stages are based on low resource levels either in the egg or during nestling feeding. The variation in ambient spring temperatures in our study area might not have been sufficient to result in large enough variation in resource levels to affect the metabolic phenotype.

These mechanisms might help the embryo to acclimatize to sub-optimal immediate thermal conditions without any positive or even detrimental consequences later in life. However, it could also be an example of adaptive transgenerational plasticity that could improve the success of the individual in the environment it will likely encounter in future life (Monaghan 2008; Ruuskanen et al. 2021). In line with this, Muscovy ducklings Cairina moschata originating from eggs incubated at a low temperature preferred lower ambient temperatures after hatching than normally incubated ducklings (Tzschentke 2008). If the temperature perceived by the embryo during incubation is indicative of the general climate during the winter, as found in this study, it could be adaptive to adjust the metabolic phenotype to these predicted conditions. Decreasing ambient temperatures may select for a higher thermogenic capacity with a concomitant increase in BMR (Swanson and Olmstead 1999). This would be beneficial in cold environments, as reflected in typical increases of BMR with latitudinal gradients (Broggi et al. 2019), with the approaching winter season (Swanson 2010), with decreasing ambient temperatures (this study) and a differential survival advantage of high-BMR blue tits in cold environments (Nilsson and Nilsson 2016). Thus, some of the inter-population variation in metabolic rates due to latitude could be set by adaptive developmental plasticity triggered during the incubation phase.

In years when winter ambient temperatures are colder or warmer than expected based on incubation temperatures, we predict a reduced recruitment rate due to a mismatch between ambient conditions and the metabolic phenotype that increases mortality during the winter. Environmental stochasticity impacting on recruitment rate has been suggested as a potential explanation for inter-specific variation in population variability (Sæther et al. 2016). A mismatch in temperatures between the incubation stage and the subsequent winter may constitute such an environmental stochasticity. One of the predictions concerning future climate change is that the unpredictability will increase (IPCC 2012) which potentially will increase the risk of a mismatch between temperatures during spring and winter, resulting in increased variation in population size.

The metabolic phenotype is flexible and can be adjusted to prevailing conditions allowing organisms to buffer changes. However, part of this flexibility seems also to be tuned by the thermal environment experienced during early life. The extent to which temperature conditions change congruently over different individual life-history episodes will determine the adaptive value of this mechanism.

\section{Declarations}

\section{Acknowledgements}


We are in debt with numerous field assistants that helped with the trapping and processing of the birds. Prof. Markku Orell and other staff from the Oulu University Research Facility provided logistic, technical support and field assistance. Dr. Jaime Potti provided essential bureaucratic help.

\section{Funding}

JB has been funded by the Andalucía Talent Hub Program launched by the Andalusian Knowledge Agency, co-funded by the European Union's Seventh Framework Program, Marie Skłodowska-Curie actions (COFUND - Grant Agreement $n^{\circ}$ 291780) and the Ministry of Economy, Innovation, Science and Employment of the Junta de Andalucía. Supported by the Academy of Finland projects no. 102286 and 47195, and the Thule Institute of the University of Oulu (JB and EH) and by grants from the Swedish Research Council no. S-AA/F0 09677-311, B 650-19981108/2000, 629-2002-1893 and 621-2003-2912 (JÅN).

\section{Author contribution statement}

JB conceived and designed the study, carried out the field work, analyzed the data and drafted the manuscript. EH, KK and SR participated in the field work, and the study design. JÅN participated in conceiving and designing the study, and in analyzing data and drafting of the manuscript.

\section{Conflicts of interest}

The authors have no conflicts of interest to declare that are relevant to the content of this article.

\section{Ethics approval}

All applicable institutional and national guidelines for the care and use of animals were followed.

\section{Availability of data and material}

Dataset analysed in this study will be available from the Digital CSIC Repository http://dx.doi.org/10.20350/digitalCSIC/12612 upon reasonable request.

\section{References}

Ardia DR, Clotfelter ED (2007) Individual quality and age affect responses to an energetic constraint in a cavity-nesting bird. Behav Ecol 18:259-266

Ardia DR, Pérez JH, Chad EK, Voss MA, Clotfelter ED (2009) Temperature and life history: experimental heating leads female tree swallows to modulate egg temperature and incubation behaviour. J Anim Ecol 78:4-13

Bachman GC, Chappell MA (1998) The energetic cost of begging in nestling house wrens. Anim Behav 55:1607-1618 
Beaman JE, White CR, Seebacher F (2016) Evolution of Plasticity: Mechanistic Link between Development and Reversible Acclimation. Trend Ecol Evol 31:237-249

Ben-Ezra N, Burness G (2017) Constant and cycling incubation temperatures have long-term effects on the morphology and metabolic rate of Japanese quail. Physiol Biochem Zool 90:96-105

Berntsen HH, Bech C (2016) Incubation temperature influences survival in a small passerine bird. J Avian Biol 47:141-145

Bouwhuis S, Sheldon BC, Verhulst S (2011) Basal metabolic rate and the rate of senescence in the great tit. Funct Ecol 25:829-838

Broggi J, Hohtola E, Koivula K, Orell M, Nilsson J-Å (2009) Long-term repeatability of winter basal metabolic rate and mass in a wild passerine. Funct Ecol 23:768-773

Broggi J, Nilsson J, Koivula K, Hohtola E, Nilsson J-Å (2019) Mass or pace? Seasonal energy management in wintering boreal passerines. Oecologia 189:339-351

Broggi J, Orell M, Hohtola E, Nilsson J- $\AA$ (2004) Metabolic response to temperature variation in the great tit: an interpopulation comparison. J Anim Ecol 73:967-972

Bushuev AV, Husby A, Sternberg H, Grinkov VG (2012) Quantitative genetics of basal metabolic rate and body mass in free-living pied flycatchers. J Zool 288:245-251

Careau V, Buttemer WA, Buchanan KL (2014) Early-developmental stress, repeatability, and canalization in a suite of physiological and behavioural traits in female zebra finches. Integr Comp Biol 54:539-554

Carey C, Dawson WR (1999) A search for environmental cues used by birds in survival of cold winters. Curr Ornithol 15:1-31

Cortés PA, Petit M, Lewden A, Milbergue M, Vézina F (2015) Individual inconsistencies in basal and summit metabolic rate highlight flexibility of metabolic performance in a wintering passerine. $\mathrm{J}$ Exp Zool 323A: $179-190$

Criscuolo F, Monaghan P, Nasir L, Metcalfe NB (2008) Early nutrition and phenotypic development: 'catch up' growth leads to elevated metabolic rate in adulthood. Proc R Soc B 275:1565-1570

Douhard M, Gaillard J-M, Delorme D, Capron G, Duncan P, Klein F, Bonenfant C (2013) Variation in adult body mass of roe deer: early environmental conditions influence early and late body growth of females. Ecology 94:1805-1814

Dubois K, Hallot F, Vézina F (2016) Basal and maximal metabolic rates differ in their response to rapid temperature change among avian species. J Comp Physiol B 186:919-935 
DuRant SE, Hopkins WA, Wilson AF, Hepp GR (2012) Incubation temperature affects the metabolic cost of thermoregulation in a young precocial bird. Funct Ecol 26:416-422

Fairlie J, Holland R, Pilkington JG, Pemberton JM, Harrington L, Nussey DH (2016) Lifelong leukocyte telomere dynamics and survival in a free-living mammal. Aging Cell 15:140-148

Forchhammer MC, Clutton-Brock TH, Lindström J, Albon SD (2001) Climate and population density induce long-term cohort variation in a northern ungulate. J Anim Ecol 70:721-729

Groothuis TGG, Müller W, von Engelhardt N, Carere C, Eising C (2005) Maternal hormones as a tool to adjust offspring phenotype in avian species. Neurosc Biobehav Rev 29:329-352

Hill RW (1972) Determination of oxygen consumption by use of a para magnetic oxygen analyzer. J Appl Physiol 33:261-263

IPCC (2012) Managing the risks of extreme events and disasters to advance climate change adaptation. A special report of working groups I and II of the intergovernmental panel on climate change. Cambridge University Press

Jenni L, Winkel R (1994) Moult and Aging of European Passerines. Academic Press, London

Jimenez AG (2018) "The same thing that makes you live can kill you in the end": Exploring the effects of growth rates and longevity on cellular metabolic rates and oxidative stress in mammals and birds. Integr Comp Biol 58:544-558

Jimenez AG, Williams JB (2014) Differences in muscle fiber size and associated energetic costs in phylogenetically paired tropical and temperate birds. Physiol Biochem Zool 87:752-761

Järvinen A (1990) Incubation and nestling periods in hole-nesting passerines in Finnish Lapland. Ornis Fennica 67:65-72

Karvonen J, Orell M, Rytkönen S, Broggi J, Belda EJ (2012) Population dynamics of an expanding passerine at the distribution margin. J Avian Biol 43:102-108

Latimer CE, Cooper SJ, Karasov WH, Zuckerberg B (2018) Does habitat fragmentation promote climateresilient phenotypes? Oikos 127:1069-1080

Lessells CM, Boag PT (1987) Unrepeatable repeatabilities: a common mistake. Auk 104:116-121

Lindström J, Kokko H (2002) Cohort effects and population dynamics. Ecol Lett 5:338-344

Loyau T, Bedrani L, Berri C, Métayer-Coustard S, Praud C, Coustham V, Mignon-Grasteau S, Duclos MJ, Tesseraud S, Rideau N, Hennequet-Antier C, Everaert N, Yahav S, Collin A (2014) Cyclic variations in incubation conditions induce adaptive responses to later exposure in chickens: a review. Animal 9:76-85 
Martin TE, Ton R, Oteyza JC (2018) Adaptive influence of extrinsic and intrinsic factors on variation of incubation periods among tropical and temperate passerines. Auk 135:101-113

Mathot KJ Martin K, Kempenaers B, Forstmeier W (2013) Basal metabolic rate can evolve independently of morphological and behavioural traits. Heredity 111:175-181

Mertens JAL (1980) The energy requirements for incubation in great tits and other bird species. Ardea 68:85-192

McKechnie AE (2008) Phenotypic flexibility in basal metabolic rate and the changing view of avian physiological diversity: a review. J Comp Physiol B 178:235-247

McKechnie AE, Chetty K, Lovegrove BG (2007) Phenotypic flexibility in basal metabolic rate in laughing doves: responses to short-term thermal acclimation. J Exp Biol 210:97-106

McKechnie AE, Swanson DL (2010) Sources and significance of variation in basal, summit and maximal metabolic rates in birds. Curr Zool 56:741-758

McNab BK (1997) On the utility of uniformity in the definition of basal rate of metabolism. Physiol Zool 70:718-720

Monaghan P (2008) Early growth conditions, phenotypic development and environmental change. Phil Trans R Soc B 363:1635-1645

Naya DE, Spangenberg L, Naya H, Bozinovic F (2013) How does evolutionary variation in basal metabolic rates arise? A statistical assessment and mechanistic model. Evolution 67:1463-1476.

Nilsson J-Å, Åkesson M, Nilsson JF (2009) Heritability of resting metabolic rate in a wild population of blue tits. J Evol Biol 22:1867-1874.

Nilsson JF, Nilsson J-Å (2016) Fluctuating selection on basal metabolic rate. Ecol Evol 6:1197-1202.

Nord A, Giroud S (2020) Lifelong effects of thermal challenges during development in birds and mammals. Front Physiol 11:419.

Nord A, Nilsson J-Å (2011) Incubation temperature affects growth and energy metabolism in blue tit nestlings. Am Nat 178:639-651.

Nord A, Nilsson J-Å (2016) Long-term consequences of high incubation temperature in a wild bird population. Biol Lett 12:20160087.

Nord A, Sandell MI, Nilsson J-Å (2010) Female zebra finches compromise clutch temperature in energetically demanding incubation conditions. Funct Ecol 24:1031-1036. 
Ojanen M (1983) Effects of laying sequence and ambient temperature on the composition of eggs of the great tit Parus major and the pied flycatcher Ficedula hypoleuca. Ann Zool Fenn 20:65-71.

Orell M, Ojanen M (1983) Timing and length of the breeding season of the great tit Parus major and the willow tit $P$. montanus near Oulu, northern Finland. Ardea 71:183-198.

Pakanen V-M, Orell M, Vatka E, Rytkönen S, Broggi J (2016) Different ultimate factors define timing of breeding in two related species. PLoS ONE 11:e0162643.

Petit M, Clavijo-Baquet S, Vézina F (2017) Increasing winter maximal metabolic rate improves intrawinter survival in small birds. Physiol Biochem Zool 90:166-177.

Petit M, Lewden A, Vézina F (2013) Intra-seasonal flexibility in avian metabolic performance highlights the uncoupling of basal metabolic rate and thermogenic capacity. PLoS ONE 8:e68292.

Petit M, Vézina F (2014a) Reaction norms in natural conditions: How does metabolic performance respond to weather variations in a small endotherm facing cold environments? PLoS ONE 9:e113617.

Petit M, Vézina F (2014b) Phenotype manipulations confirm the role of pectoral muscles and haematocrit in avian maximal thermogenic capacity. J Exp Biol 217:824-830.

Reed WL, Clark ME (2011) Beyond maternal effects in birds: responses of the embryo to the environment. Integr Comp Biol 51:73-80.

Reid JM, Bignal EM, Bignal S, McCracken DI, Monaghan P (2003) Environmental variability, life-history covariation and cohort effects in the red-billed chough Pyrrhocorax pyrrhocorax. J Anim Ecol 72:36-46.

Reinertsen RE, Haftorn S (1986) Different metabolic strategies of northern birds for nocturnal survival. J Comp Physiol B 156:655-663.

Rønning B, Broggi J; Bech C, Moe B, Ringsby TH, Pärn H, Hagen IJ, Saether BE, Jensen H (2015) Is basal metabolic rate associated with recruit production and survival in free-living house sparrows? Funct Ecol 30:1140-1148

Rønning B, Jensen H, Moe B, Bech C (2007) Basal metabolic rate: heritability and genetic correlations with morphological traits in the zebra finch. J Evol Biol 20:815-1822.

Rose KE, Clutton-Brock TH, Guinness FE (1999) Cohort variation in male survival and lifetime breeding success in red deer. J Anim Ecol 67:979-986.

Ruuskanen S, Hsu B-Y, Nord A (2021) Endocrinology of thermoregulation in birds in a changing climate. Mol Cell Endocrin 519:111088.

Sheldon EL, Schrey AW, Hurley LL, Griffith SC (2020) Dynamic changes in DNA methylation during postnatal development in zebra finches Taeniopygia guttata exposed to different temperatures. J Avian 
Biol e02294.

Swanson DL (2010) Seasonal metabolic variation in birds: functional and mechanistic correlates Curr Ornithol 17:75-129.

Swanson DL, McKechnie AE, Vézina F (2017) How low can you go? An adaptive framework for interpreting basal metabolic rate variation in endotherms. J Comp Physiol B 187:1039-1056.

Swanson DL, Olmstead KL (1999) Evidence for a proximate influence of winter temperature on metabolism in passerine birds. Physiol Biochem Zool 72:566-575.

Sæther B-E et al. (2016) Demographic routes to variability and regulation in bird populations. Nature Comm. 7:12001.

Thomson DL, Monaghan P, Furness RW (1998) The demands of incubation and avian clutch size. Biol Rev 73:293-304.

Tieleman BI, Versteegh MA, Fries A, Helm B, Dingemanse NJ, Gibbs HL, Williams JB (2009). Genetic modulation of energy metabolism in birds through mitochondrial function. Proc R Soc B 276:1685-1693.

Tzschentke B (2008) Monitoring the development of thermoregulation in poultry embryos and its influence by incubation temperature. Comput Electron Agr 64:61-71.

Valkama J, Vepsäläinen V, Lehikoinen A (2011) The Third Finnish Breeding Bird Atlas. Finnish Museum of Natural History and Ministry of Environment. <http://atlas3.lintuatlas.fi/english> ISBN 978-952-10-7145-4

Vatka E, Orell M, Rytkönen S (2016) The relevance of food peak architecture in trophic interactions. Glob Chang Biol 22:1585-1594.

Verhulst S, Holveck M-J, Riebel K (2006) Long-term effects of manipulated natal brood size on metabolic rate in zebra finches. Biol Lett 2:478-480.

Versteegh MA, Helm B, Dingemanse NJ, Tieleman BI (2008) Repeatability and individual correlates of basal metabolic rate and total evaporative water loss in birds: A case study in European stonechats. Comp Biochem Physiol A 150:452-457.

Vézina F, Gustowska A, Jalvingh KM, Chastel O, Piersma T (2009) Hormonal correlates and thermoregulatory consequences of molting on metabolic rate in a northerly wintering shorebird. Physiol Biochem Zool 82:129-142.

Wada H, Kriengwatana B, Allen N, Schmidt KL, Soma KK, MacDougall-Shackleton SA (2015) Transient and permanent effects of suboptimal incubation temperatures on growth, metabolic rate, immune function and adrenocortical responses in zebra finches. J Exp Biol 218:2847-2855.

West-Eberhard MJ (2003) Developmental plasticity and evolution. Oxford University Press. 
Wiersma P, Nowak B, Williams JB (2012) Small organ size contributes to the slow pace of life in tropical birds. J Exp Biol 215:1662-1669.

Zheng W-H, Li M, Liu J-S, Shao S-L, Xu X-J (2014a) Seasonal variation of metabolic thermogenesis in Eurasian tree sparrows (Passer montanus) over a latitudinal gradient. Physiol Biochem Zool 87:704-718.

Zheng W-H, Liu J-S, Swanson DL (2014b) Seasonal phenotypic flexibility of body mass, organ masses, and tissue oxidative capacity and their relationship to resting metabolic rate in Chinese Bulbuls. Physiol Biochem Zool 87:432-444.

Zuur AF leno EN, Elphick CS (2010) A protocol for data exploration to avoid common statistical problems. Meth Ecol Evol1:3-14.

\section{Figures}

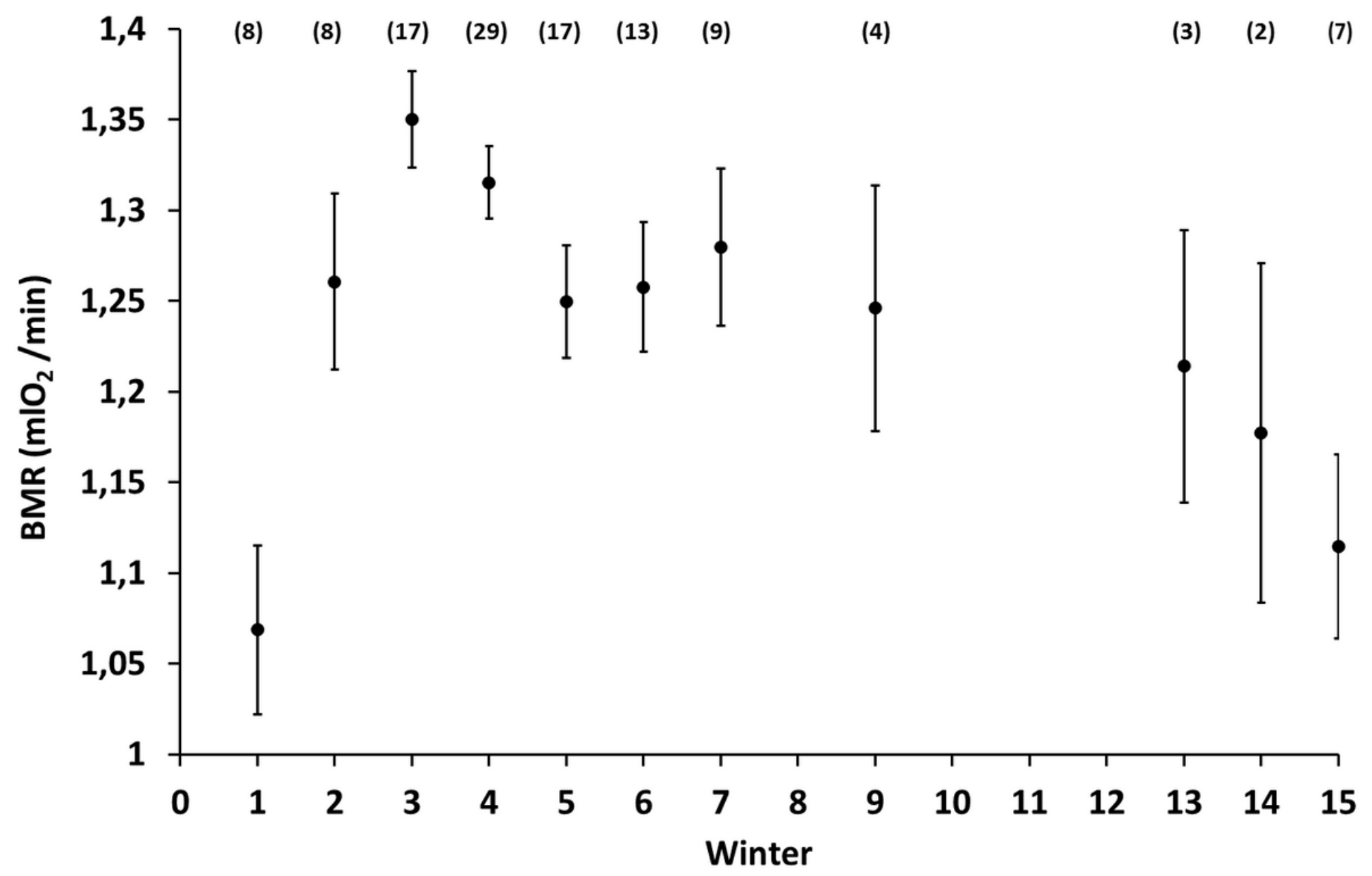

\section{Figure 1}

Least square means $( \pm S E)$ of BMR of first-year great tits during 11 winters when standardized for the factors in Table 1, i.e. date, minimum temperature and body mass. Winter 1 denotes the winter 19992000. Numbers in parentheses denotes sample size. 


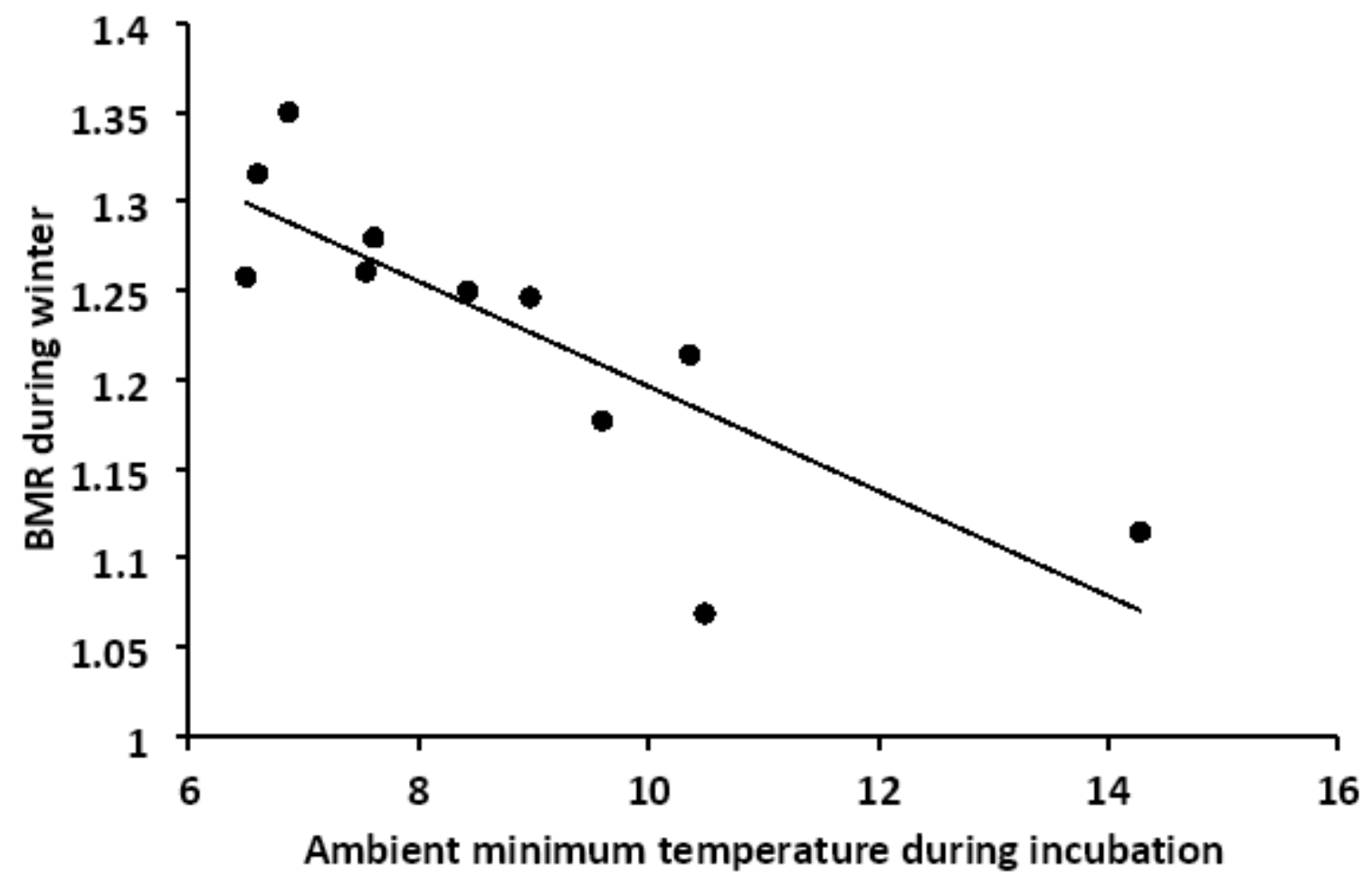

Figure 2

Average winter BMR for first-year great tits, standardized by date, average minimum temperatures the week before capture and body mass at capture, i.e. corrected for the model presented in Table 1. Winter estimates of BMR for first-year birds are presented in relation to minimum temperatures experienced during the incubation period in the previous breeding season $(F 1,9=17.9, P=0.0022)$. Equation of the line: $\mathrm{BMR}=1.49-0.029 \times$ Temp.

\section{Supplementary Files}

This is a list of supplementary files associated with this preprint. Click to download.

- ESMBMRlongtermOEC.docx 